\section{Essay}

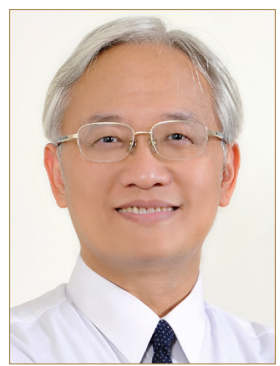

\section{Corresponding Author}

Wen-Cheng Huang

(iD https://orcid.org/0000-0003-4249-710X

Department of Neurosurgery, Neurological Institute, Taipei Veterans General Hospital, Room 525, 17F, No. 201, Shih-Pai Road,

Sec. 2, Beitou, Taipei 11217, Taiwan

Tel: +886-2-28757718

Fax: +886-2-28757702

E-mail:wchuang518@gmail.com

Received: September 2, 2018

Revised: October 24, 2018

Accepted: October 28, 2018

\title{
Taiwan Neurosurgical Spine Society: The New Shining Star
}

\author{
Yi-Hsuan $\mathrm{Kuo}^{1,2}$, Jau-Ching $\mathrm{Wu}^{1,2}$, Wen-Cheng Huang ${ }^{1,2}$, Ming-Chao Huang ${ }^{1}$, \\ E-Jian Lee ${ }^{3}$, Henrich Cheng ${ }^{1,2,4}$ \\ ${ }^{1}$ Department of Neurosurgery, Neurological Institute, Taipei Veterans General Hospital, Taipei, Taiwan \\ ${ }^{2}$ School of Medicine, National Yang-Ming University, Taipei, Taiwan \\ ${ }^{3}$ Neurosurgical Service, Department of Surgery, National Cheng Kung University Hospital, College of \\ Medicine, National Cheng Kung University, Tainan, Taiwan \\ ${ }^{4}$ Institute of Pharmacology, National Yang-Ming University, Taipei, Taiwan
}

As spine surgery flourished in Taiwan and neurosurgeons became more involved in spine surgery towards the end of the 20th century, the Taiwan Neurosurgical Spine Society (TNSS), earlier named the Taiwan Neurospinal Society, was established on March 11, 2001. As its main founder, Dr. Chun-I Huang was elected as the first president of the TNSS. The goals of the TNSS were to promote research, to hold academic seminars, to participate in international conferences, and to exchange clinical experiences. The mission of the TNSS was successful, and the profession of spine surgery in Taiwan advanced during the first decade of the 21st century, culminating in the TNSS joining ASIA SPINE in 2010. Since its establishment, the TNSS has always been supportive of collaboration and communication with the Korean Spinal Neurosurgery Society and the Neurospinal Society of Japan. Through periodical meetings, supported by the TNSS, surgeons worldwide have enjoyed a platform of sharing and mutual learning. To further promote academic research, the TNSS has officially supported the journal Neurospine since 2018. With extensive efforts from local and international surgeons, the TNSS will continue to adhere to its mission and to advance the profession of spine surgery.

Keywords: Taiwan Neurosurgical Spine Society, Spine surgery

\section{INTRODUCTION}

Taiwan, located in East Asia and the West Pacific, near Korea and Japan, is a beautiful island full of nature scenes and cultural legacies. The total area of Taiwan proper and its outlying islands is around 36,197 square kilometers. Around 20 percent of the area is protected, and Jade Mountain is the highest peak in East
Asia. The National Palace Museum in Taipei City contains one of the largest collections of ancient Chinese artifacts in the world. ${ }^{1}$ Taiwan is also well-known worldwide for its healthcare systemthe National Health Insurance (NHI) scheme. The NHI, instituted in March 1995, is a government-implemented social insurance scheme, with a single-payer system and global budget payment system. The NHI coverage rate is $99.7 \%$. The total 
healthcare expenditure accounts for about $6.1 \%$ of Gross Domestic Product, and slightly more than half of it was spent on the NHI. ${ }^{2}$
Among the 23 million Taiwanese there are more than 46,000 physicians - that is, 19.71 per ten thousand persons-and 532 practicing board-certified neurosurgeons; women account for

Table 1. Neurosurgery training hospitals in Taiwan, 2018

\begin{tabular}{|c|c|}
\hline Major training hospitals $(\mathrm{n}=19)$ & Cooperative hospitals $(n=10)$ \\
\hline National Taiwan University Hospital & Taipei City Hospital-Duties-Renai Branch \\
\hline Taipei Veterans General Hospital & Far Eastern Memorial Hospital \\
\hline Tri-Service General Hospital & Shin Kong Wu Ho-Su Memorial Hospital \\
\hline Mackay Memorial Hospital & Taipei Municipal Wanfang Hospital \\
\hline Cathay General Hospital & Keelung Chang Gung Memorial Hospital \\
\hline Taipei Medical University Hospital & Chung Shan Medical University Hospital \\
\hline Taipei Medical University-Shuang Ho Hospital & Tungs' Taichung MetroHarbor Hospital \\
\hline Linkou Chang Gung Memorial Hospital & Tainan Municipal An-Nan Hospital \\
\hline Taichung Veterans General Hospital & Taipei Tzu Chi Hospital \\
\hline China Medical University Hospital & Taichung Tzu Chi Hospital \\
\hline \multicolumn{2}{|l|}{ Changhua Christian Hospital } \\
\hline \multicolumn{2}{|l|}{ Chiayi Chang Gung Memorial Hospital } \\
\hline \multicolumn{2}{|l|}{ National Cheng Kung University Hospital } \\
\hline \multicolumn{2}{|l|}{ Chi Mei Hospital } \\
\hline \multicolumn{2}{|c|}{ Kaohsiung Medical University Chung-Ho Memorial Hospital } \\
\hline \multicolumn{2}{|l|}{ Kaohsiung Veterans General Hospital } \\
\hline \multicolumn{2}{|l|}{ Kaohsiung Chang Gung Memorial Hospital } \\
\hline \multicolumn{2}{|l|}{ E-Da Hospital } \\
\hline Hualien Tzu Chi Hospital & \\
\hline
\end{tabular}

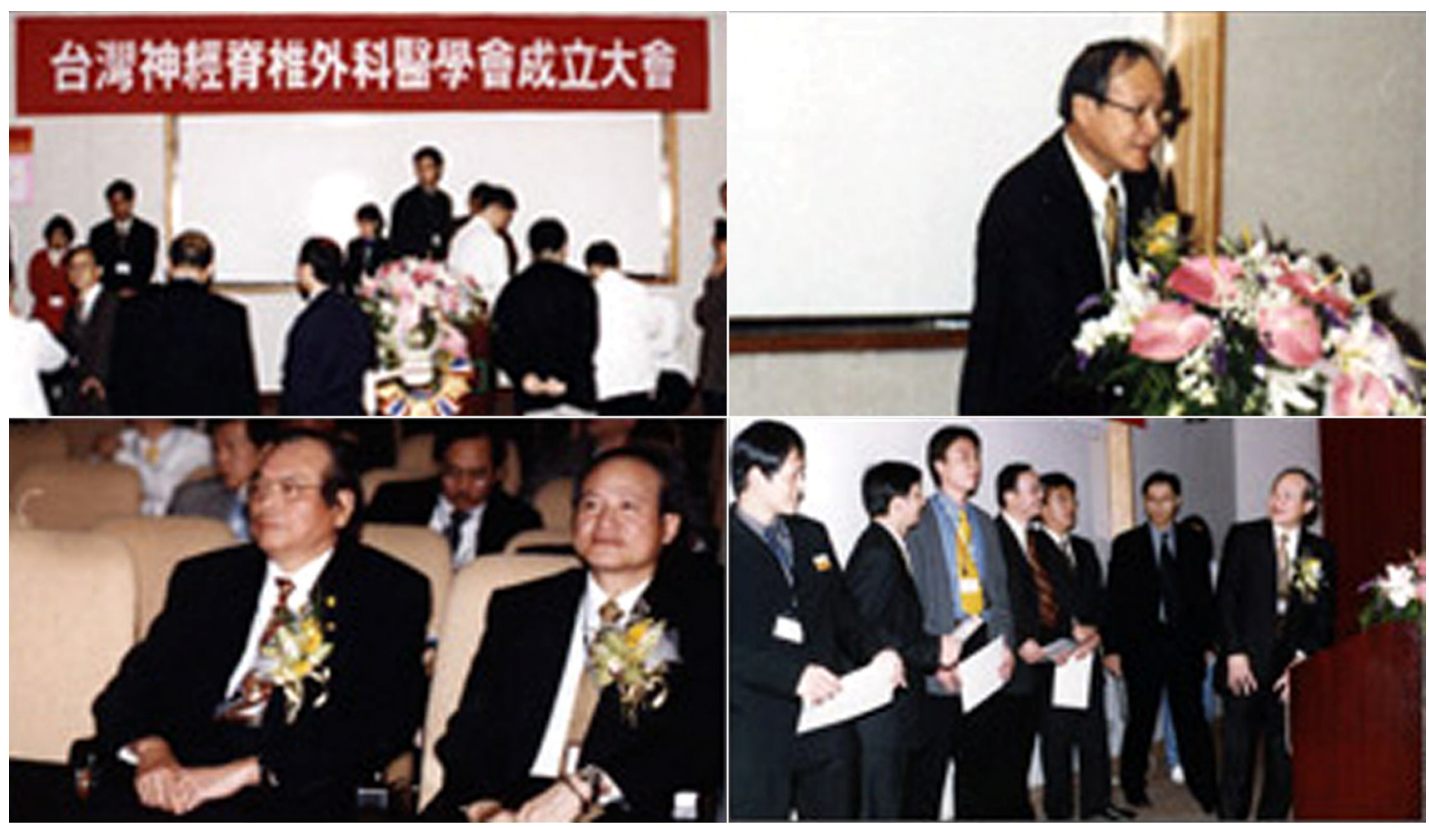

Fig. 1. The inaugural meeting of the Taiwan Neurosurgical Spine Society on March 11, 2001. 
$19.1 \%$ and $3.6 \%$, respectively. ${ }^{3}$ Neurosurgery is one of the 23 medical specialties certified by the Ministry of Health and Welfare. The development of neurosurgery in Taiwan began in the late 1950s, but it was not until 1993 that the Taiwan Neurosurgical Society was officially established. ${ }^{4}$ To become a neurosurgeon in Taiwan takes 6 years for a graduated physician to finish the residency after the postgraduate training. Under the system of a Residency Review Committee, 19 hospitals are major training hospitals and 10 others are cooperative hospitals (Table 1). These 29 hospitals together are responsible for the training program of 26 residents each year. ${ }^{5}$ During the 6-year training program, each resident is required to learn a number of topics, including neurocritical care, functional neurosurgery, spine surgery, vascular surgery, pediatric neurosurgery, and skull base surgery. Cross training in neurology, neuroradiology and neuropathology are also covered.

\section{THE ESTABLISHMENT}

As spine surgery flourished in Taiwan-for example, there were 19,385 anterior cervical discectomy and fusion surgeries from 1997 to 2007 according to the National Health Insurance Research Database ${ }^{6}$-it became abundantly clear for the need of a communicating medium between spine surgeons in order to share their experiences and for academic and scientific publishing. There was one such society about spine surgery formed in the 1990s, but which was unfortunately limited to orthope- dics. As neurosurgeons engaged more and had greater achievements in spine surgery, Dr. Chun-I Huang decided to convene neurosurgeons devoted to spine issues for our own organization in Taiwan. The assemblage began in 2000, and after months of preparation and arrangements the inaugural meeting for this organization was held in Taipei on March 11, 2001 (Fig. 1). The name "Taiwan Neurospinal Society" was agreed upon, although it was later changed to the "Taiwan Neurosurgical Spine Society" (TNSS) in 2013. Dr. Chun-I Huang was elected as the first and founding President and Dr. Ming-Chao Huang as the first Secretary.

After completing his 2-year presidency in 2003, Dr. Chun-I Huang continued to serve the society as an Honorary President

Table 2. The former and present presidents and secretaries of the Taiwan Neurosurgical Spine Society

\begin{tabular}{lrll}
\hline Term & \multicolumn{1}{c}{ Date } & \multicolumn{1}{c}{ President } & \multicolumn{1}{c}{ Secretary } \\
\hline First & $2001.3-2003.6$ & Chun-I Huang & Ming-Chao Huang \\
Second & $2003.6-2005.6$ & Chung-Long Hung & Shiuh-Lin Hwang \\
Third & $2005.6-2007.6$ & Cheng-Hsing Kao & Chao-Hung Yeh \\
Fourth & $2007.6-2009.6$ & Henrich Cheng & Wen-Cheng Huang \\
Fifth & $2009.6-2011.6$ & Yung-Hsiao Chiang & Hsin-I Ma \\
Sixth & $2011.6-2013.6$ & Shiuh-Lin Hwang & Kung-Shing Lee \\
Seventh & $2013.6-2015.6$ & Ming-Chao Huang & Jau-Ching Wu \\
Eighth & $2015.6-2017.6$ & Dar-Ming Lai & Da-Tong Ju \\
Ninth & $2017.6-C u r r e n t$ & E-Jian Lee & Ting-Chung Wang \\
\hline
\end{tabular}

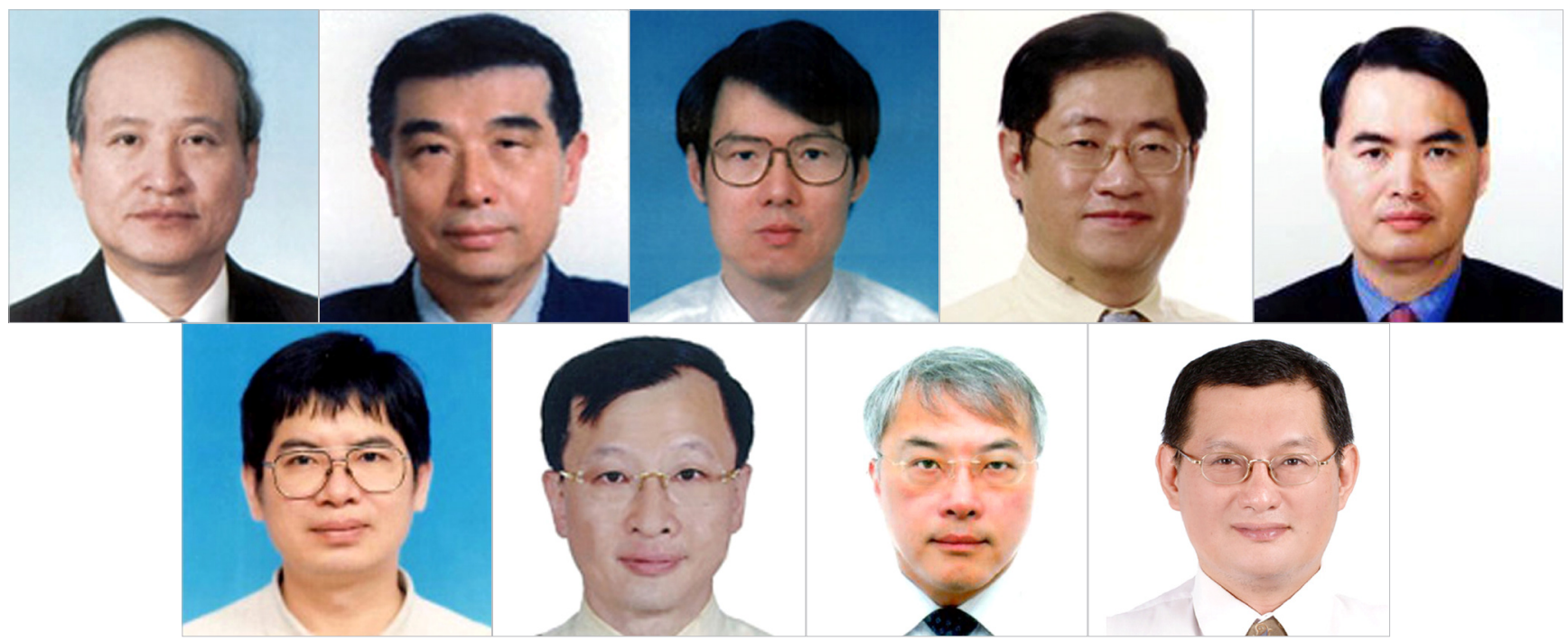

Fig. 2. The former and present presidents of the Taiwan Neurosurgical Spine Society. In order from the upper-left: Chun-I Huang, Chung-Long Hung, Cheng-Hsing Kao, Henrich Cheng, Yung-Hsiao Chiang, Shiuh-Lin Hwang, Ming-Chao Huang, Dar-Ming Lai, E-Jian Lee. 


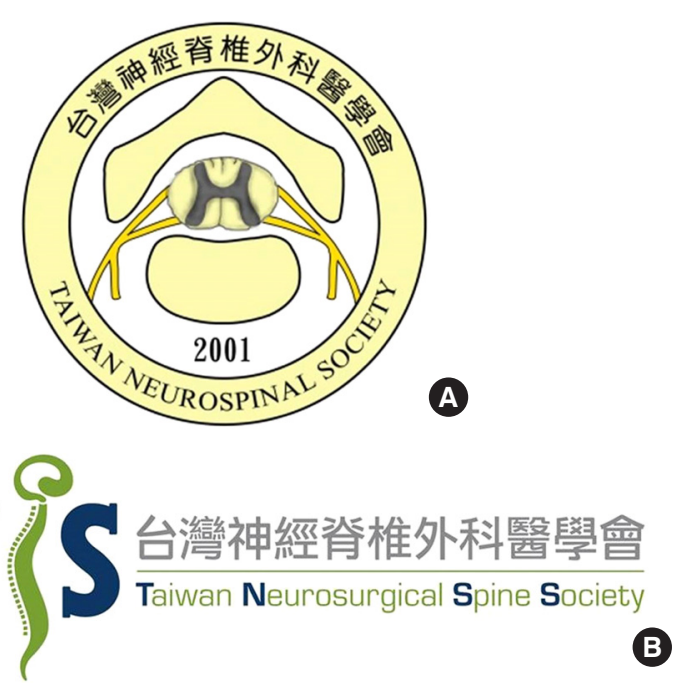

Fig. 3. The first (A) and second (B) editions of the Society's logo.

till now. Dr. Chung-Long Hung was elected as the second President, followed by Cheng-Hsing Kao, Henrich Cheng, Yung-Hsiao Chiang, Shiuh-Lin Hwang, Ming-Chao Huang, Dar-Ming Lai, and E-Jian Lee (Table 2, Fig. 2). The TNSS continued to grow under the leadership of these Presidents.

\section{VISION AND MISSION}

Our vision is to become the driving force of all spine issues in Taiwan. The TNSS is incorporated with the following 6 major goals:

(1) Research: We aim to promote both clinical and basic research about neurosurgery and spine surgery.

(2) Meetings: The annual meeting is a platform for sharing innovations and the latest research in the treatment of spinal disorders. There are also irregular academic lectures and seminars.

(3) Cooperation: We participate in international conferences and medical events to promote the exchange of relevant academic groups.

(4) Academics: Publications about nerve and spine issues in journals and books are encouraged.

(5) Education: We provide educational programs about medical practice, surgical techniques and novel innovations for experience, exchange and continuing education.

(6) Activities: Regular spine-related activities of different areas are held to achieve communication among hospitals and districts in Taiwan.

\section{THE LOGO}

The first edition of the Society's logo (Fig. 3A) was designed in 2001 and was simply the illustration of the spine axial view and the name of our Society. President Ming-Chao Huang changed it to the second edition (Fig. 3B) in 2013, in which the silhouette of the brain and spine were designed to represent the capitation " $\mathrm{S}$ " of the spine. The streamlined brain and spine surrounded by sharp characters also demonstrated the concept of soft nerve tissue covered by hard bony structures. The name was changed from "Taiwan Neurospinal Society" to "Taiwan Neurosurgical Spine Society" at the same time.

\section{THE DEVELOPMENT OF SPINE SURGERY}

At the time when the TNSS was established, neurosurgeons in Taiwan had limited ways to operate on the spine. For the cervical spine, there was anterior cervical discectomy and fusion with autograft and laminectomy. As for the lumbar spine, laminotomy, laminectomy, discectomy, interbody fusion and posterolateral fusion with autograft were performed by neurosurgeons, and there was occasionally posterior lumbar interbody fusion combined with orthopedics for pedicle screw insertion. Later on came the concept of "stabilization;" pedicle screws were inserted by Taiwan surgeons and cages were introduced to Taiwan. Cages were implanted both posteriorly and anteriorly.

President Henrich Cheng brought in the idea of "motion preservation," which consisted of: an interspinous process device, a dynamic stabilizing screw, and the artificial disc. The first lumbar artificial disc was implanted in 2005, and the first article with a large case series about cervical artificial discs in Taiwan was published in 2011. ${ }^{7}$ Following was the notion of "sagittal balance" and the popularization of standard full-length anteroposterior and lateral radiographs. Scoliosis surgery became feasible with the concept of spinal parameters. After that was the thought of "minimal invasive" surgery, including vertebroplasty, kyphoplasy, nucleoplasty, radiofrequency ablation, and the evolution of transforaminal lumbar interbody fusion (TLIF) from open, mini-open to minimally-invasive surgery. Robotics or neuro-navigation made the precise positioning of screws and minimal blood loss possible. Atlantoaxial surgery also changed a lot. In the beginning there was clamping and wiring, followed by transarticular screws, and then lateral mass screws with pedicle screws, pars screws or lamina screws.

Besides the development of surgical techniques and instrumentations, there was a notable innovation of a hemostatic ma- 

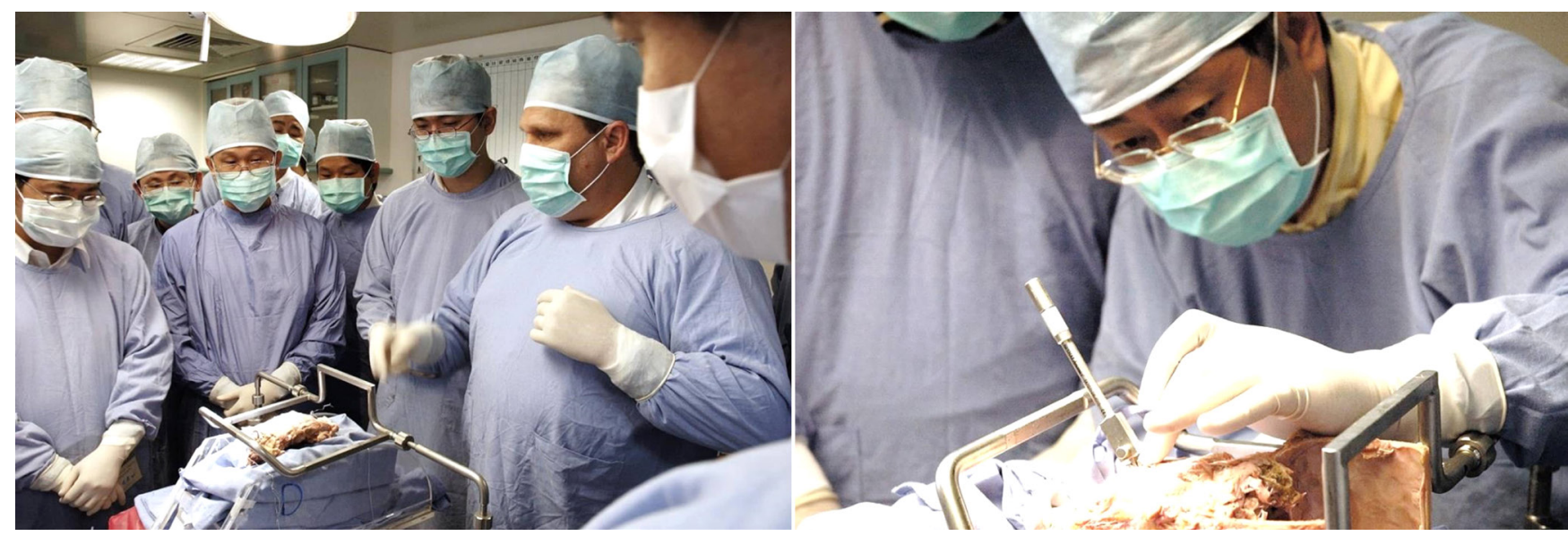

Fig. 4. Artificial cervical disc symposium and cadaver workshop in 2006.

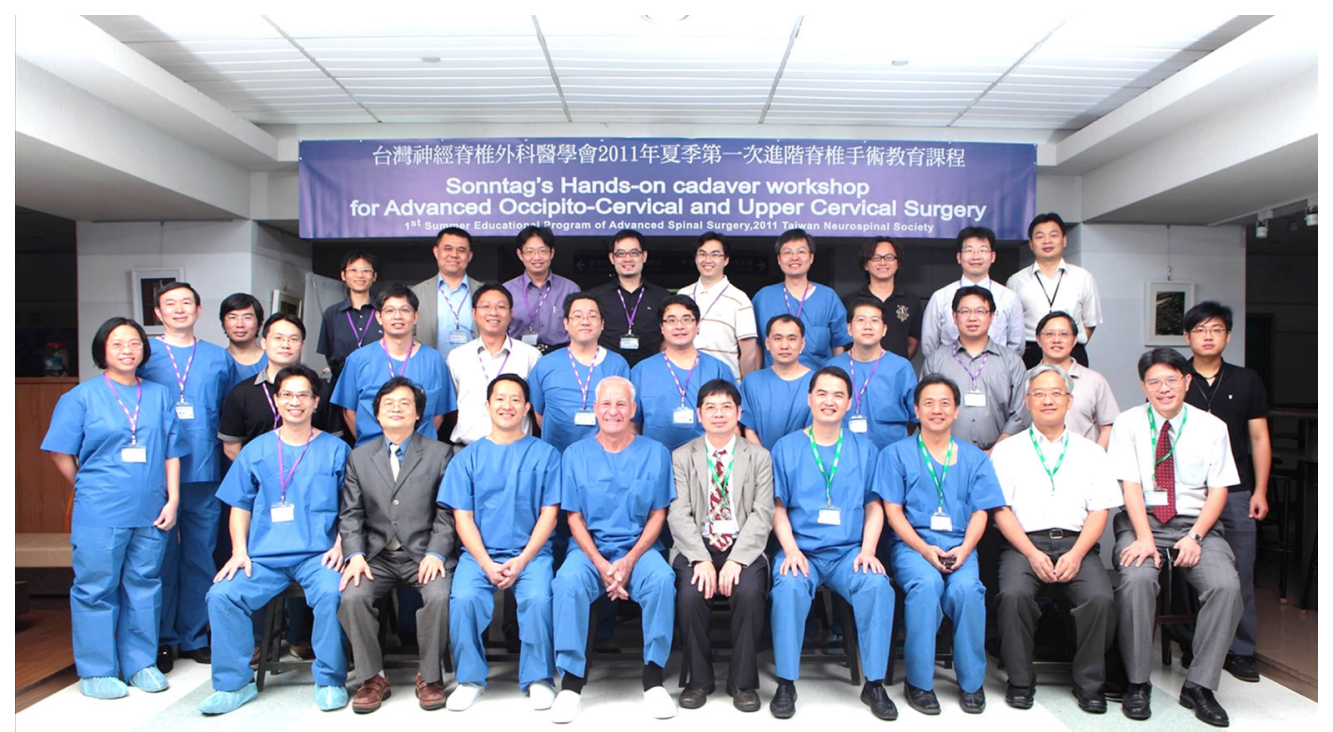

Fig. 5. Sonntag's hands-on cadaver workshop for advanced occipito-cervical and upper cervical surgery in 2011.

trix. It used to take a good deal of time for hemostasis, especially when operating at the atlantoaxial junction or ossification of posteriori longitudinal ligament. With the use of the hemostatic matrix, time was saved, bleeding was less, and therefore the safety of the operation was better.

\section{EDUCATIONAL PROGRAM}

During the development of spine surgery in Taiwan, there was always a place for the TNSS to participate. Many spine surgeons visited experts all around the world to bring novel technologies and techniques back to Taiwan. To share the knowledge, the TNSS held many symposiums, seminars and workshops for members. Some of the most notable were the work- shop for spinal cord stimulation in 2006, cervical artificial discs in 2006 and 2009 (Fig. 4), osteoporosis in 2008, polyetheretherketone rod in 2008, TLIF in 2009, and craniocervical junction and axiao-atlantic surgery in 2011 (Fig. 5); Dr. Wen-Cheng Huang ever visited the Barrow Neurological Institute to study with Professor Volker K. H. Sonntag, a pioneer in spinal neurosurgery and the 2001 President of the North American Spine Society (NASS), and Professor Sonntag was invited to Taiwan to instruct this educational program.

The TNSS also holds seasonal seminars in different areas of Taiwan for neurosurgeons to share their experiences and to communicate between each hospital. The annual meetings provide a platform to exchange opinions of clinical practice and to promote academic research in treating spinal disorders. Ever since 
Professor Henrich Cheng's study of spinal cord repair published in Science in 1996, ${ }^{8}$ neurosurgeons in Taiwan have continued to dedicate themselves to research and to publish in international journals. Dr. Jau-Ching Wu has published more than 100 manuscripts in peer-reviewed journals and was elected to the Editorial Board of the Journal of Neurosurgery: Spine in $2017 ;^{9}$ he has encouraged young neurosurgeons to follow his path in academic research.

Board-certified neurosurgeons have an opportunity to be accredited with the Taiwan Neurosurgical Spine Specialist Certification as they become TNSS members, to practice in the certified hospitals, and to have either oral presentations or posters at our annual meetings.

For residents, the TNSS is in charge of the spine section of the annual neurosurgeon residency education training program, under the instruction of the Taiwan Neurosurgical Society. The 2-day training program includes lectures from experienced spine surgeons and case presentations by residents who are asked to be the main operators or the first assistants of the surgery.

\section{ACADEMIC CONFERENCES AND INSTITUTIONAL COOPERATION}

Besides our own seasonal seminars and annual meetings, the TNSS has close interaction with the Taiwan Neurosurgical Society and societies of other subspecialties. We have many op- portunities to cooperate with societies of pain and osteoporosis, which are 2 of the most common problems encountered by patients with any kind of spine disorder. Furthermore, we hold conferences in collaboration with many other organizations (Fig. 6).

There are three major societies dealing with spine surgery in Taiwan: the TNSS, which was established purely for neurosurgeons, the Taiwan Spine Society (TWSS), which is composed only of orthopedics, and the Taiwan Society of Minimally Invasive Spine Surgery (TSMISS), in which both neurosurgeons and orthopedics participate. For better communication among them, the Taiwan Spine Summit Forum was held by these 3 societies together in January 2015 (in Taichung, president: MingChao Huang). The first joint symposium was held in February 2018 (in Tainan, president: E-Jian Lee), and we are looking forward for further deeper cooperation in the future.

The TNSS also has worked with organizations outside Taiwan. For example, the first Chinese Conference on Spine in June 2007 (in Tainan, president: Cheng-Hsing Kao) and the 7th Conferences of Taiwan and Fujian Department of Neurosurgery in June 2012 (in Kaohsiung, president: Shiuh-Lin Hwang) took up the trends of cross-strait exchanges.

The TNSS is one of the 3 member organizations of ASIA SPINE, which is the largest spine-related society for neurosurgeons in East Asia, founded in September 2010. ${ }^{10}$ The annual meeting of ASIA SPINE takes place by turns in Korea (by the Korean Spi-

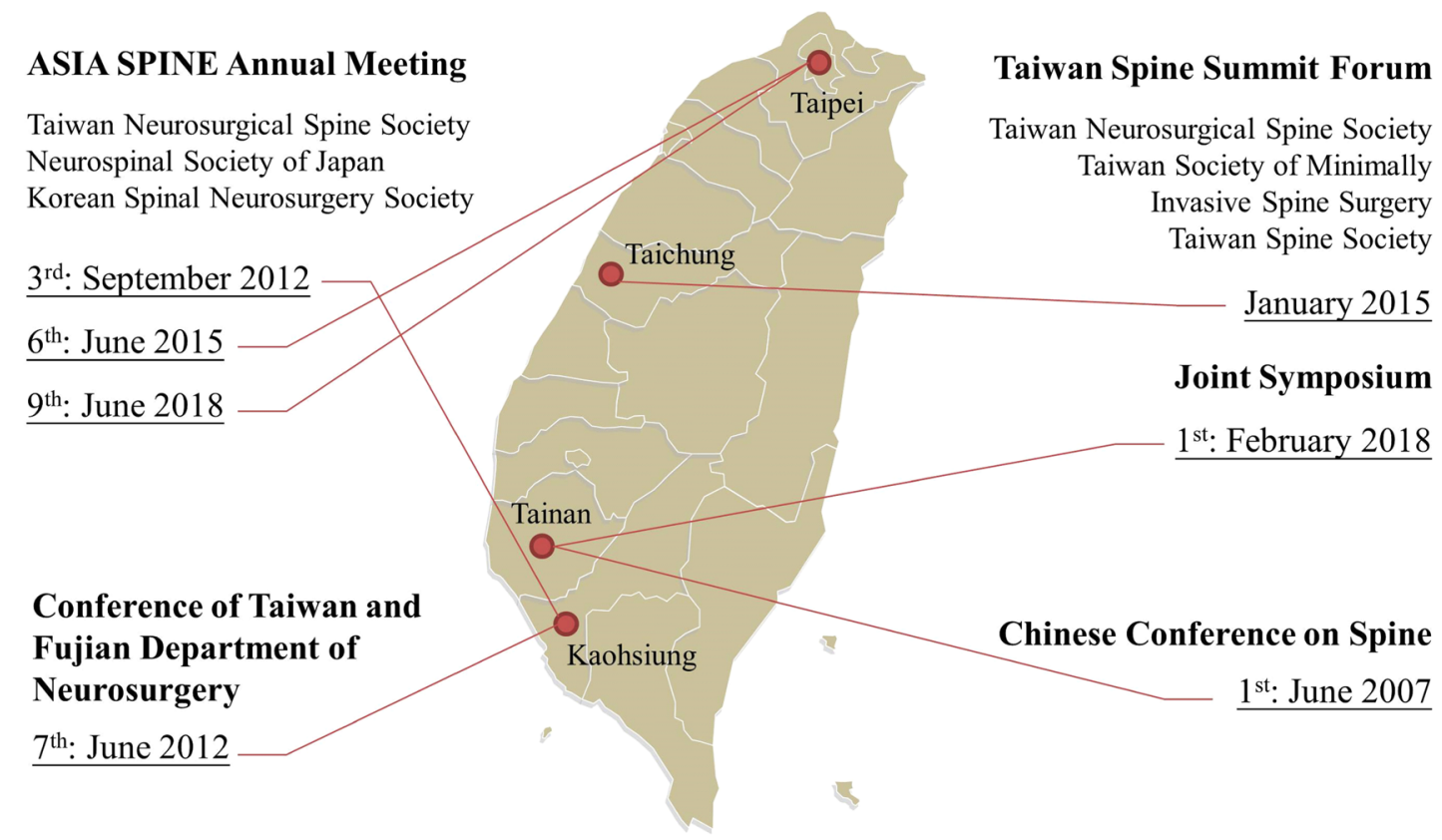

Fig. 6. Combined conferences held by the Taiwan Neurosurgical Spine Society. 
nal Neurosurgery Society), Japan (by the Neurospinal Society of Japan), and Taiwan (by the TNSS). The TNSS was responsi- ble for the 3rd meeting in September 2012 (in Kaohsiung, president: Cheng-Hsing Kao), the 6th in June 2015 (in Taipei, presi-

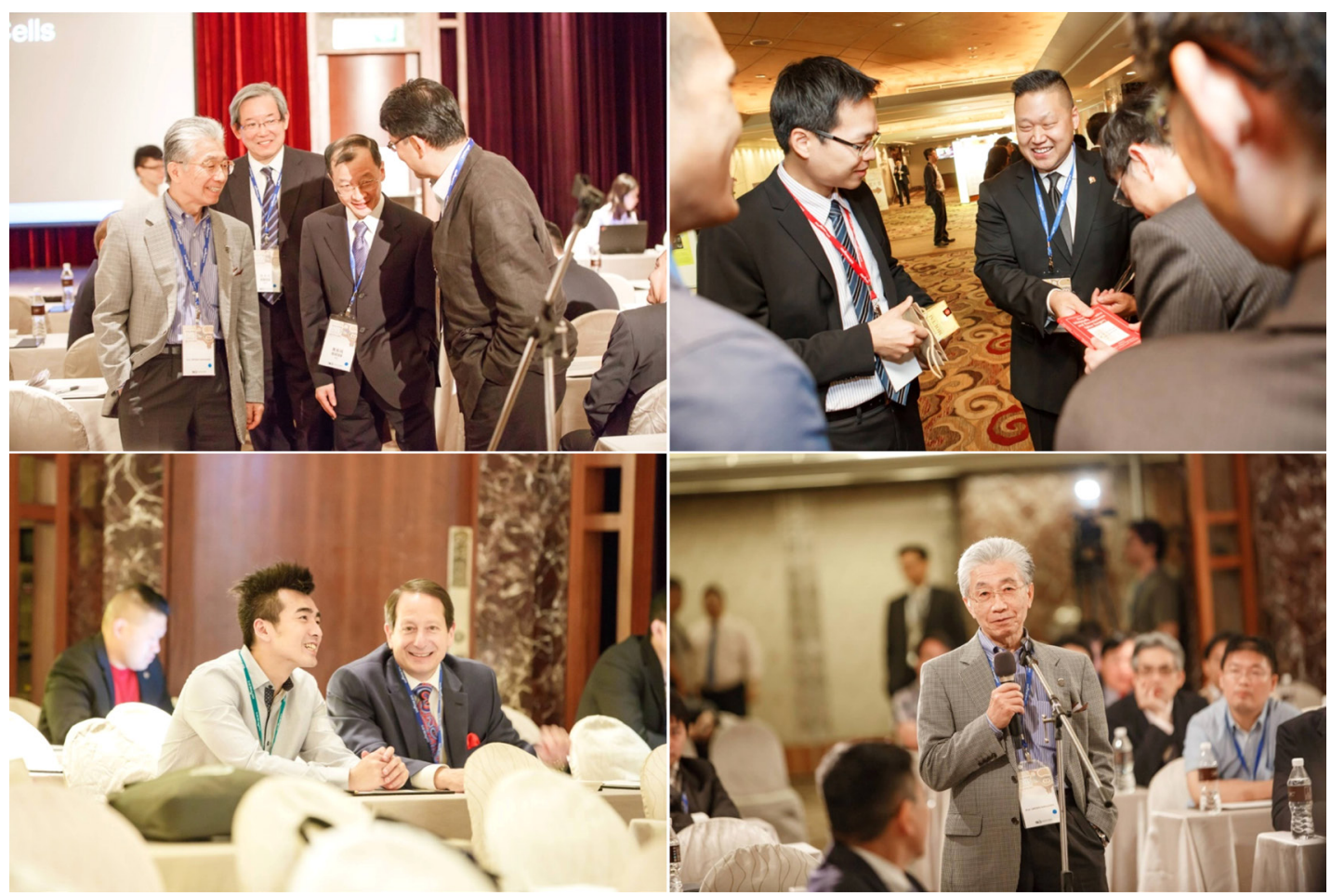

Fig. 7. ASIA SPINE, 2015.

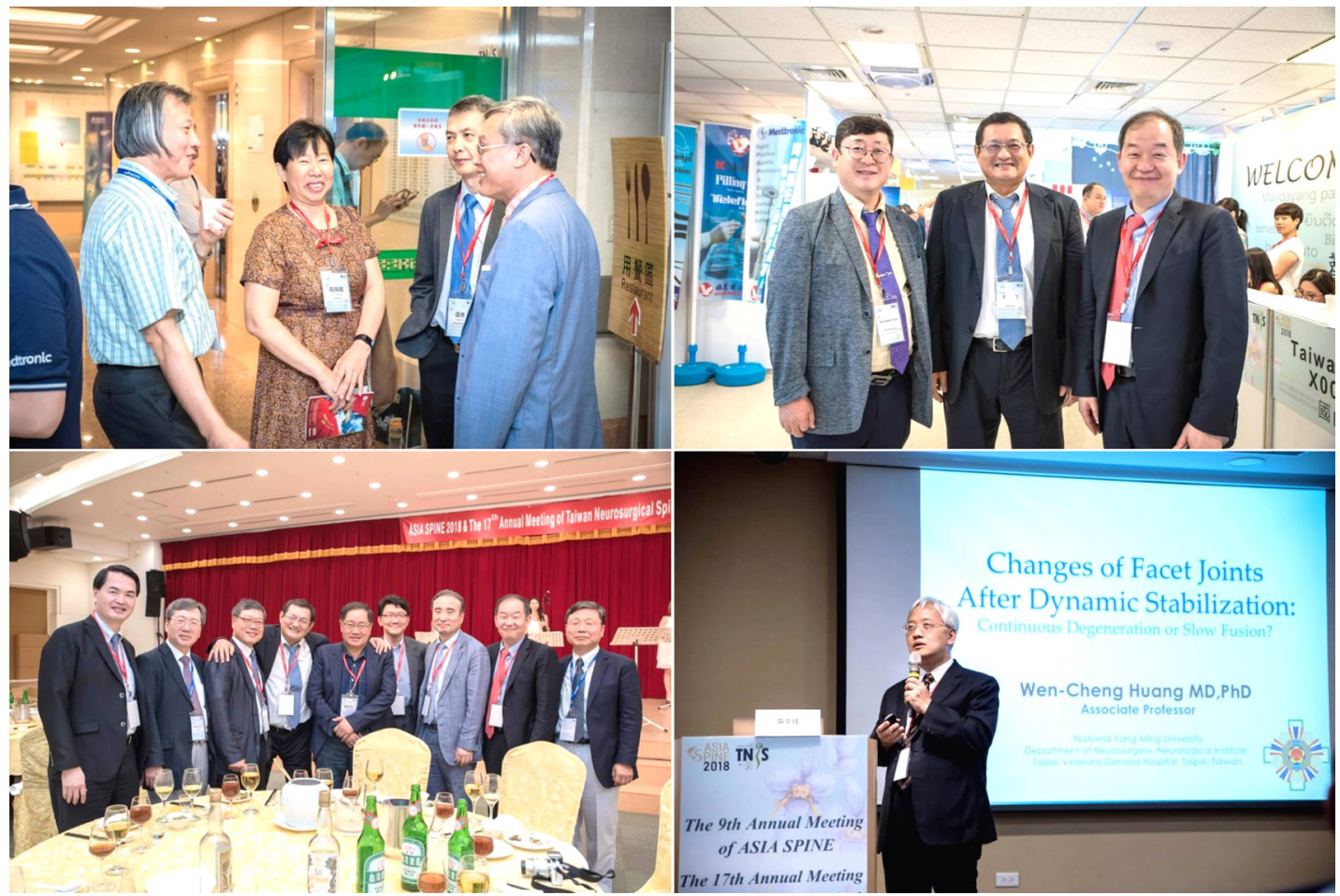

Fig. 8. ASIA SPINE, 2018. 
dent: Ming-Chao Huang), and the 9th meeting in June 2018 (in have many experts in East Asia coming to Taiwan for commuTaipei, president: E-Jian Lee) (Figs. 7, 8). It was our pleasure to nication and academic exchange, and to provide our members

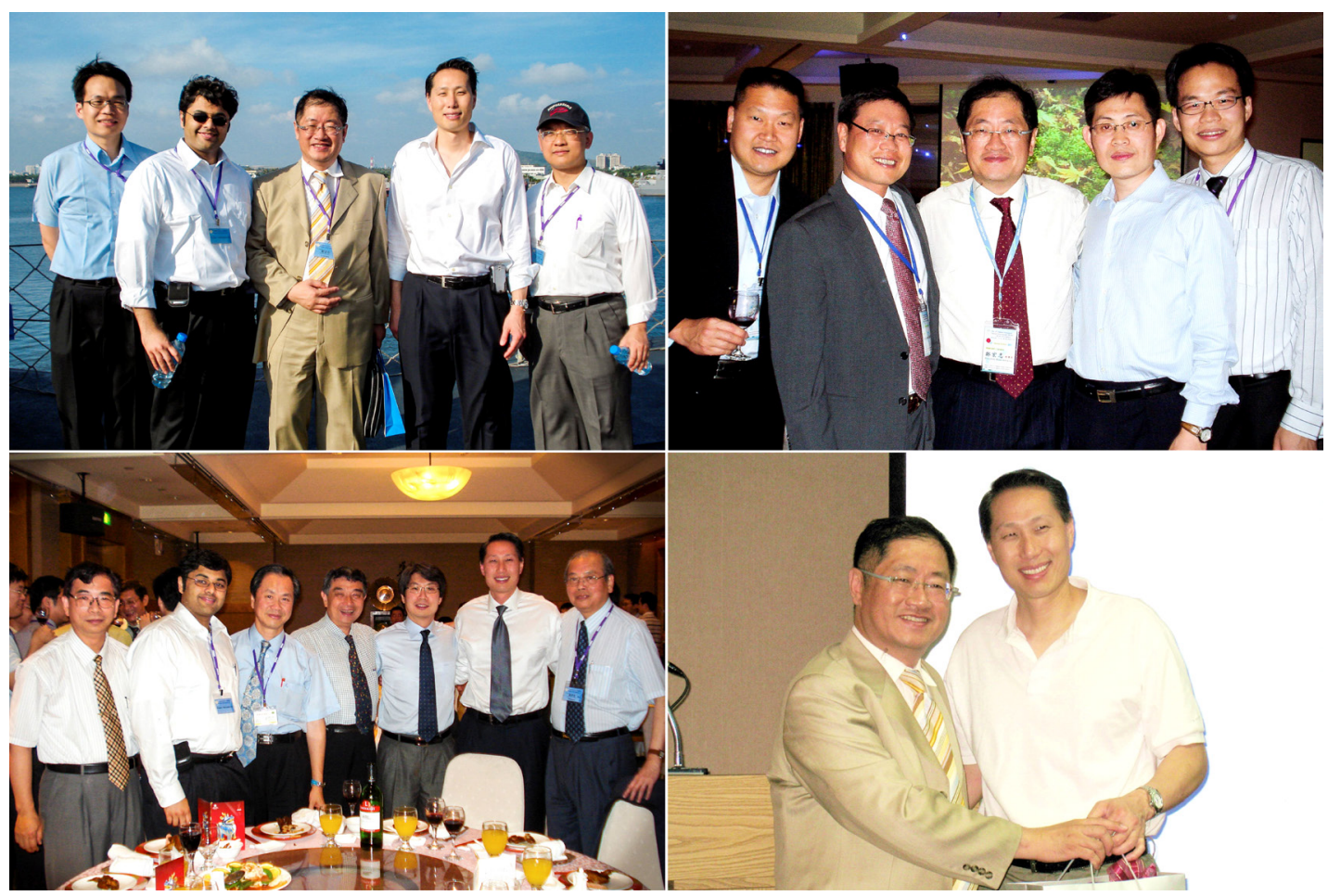

Fig. 9. Guests in 2008 and 2009 annual meetings of Taiwan Neurosurgical Spine Society.

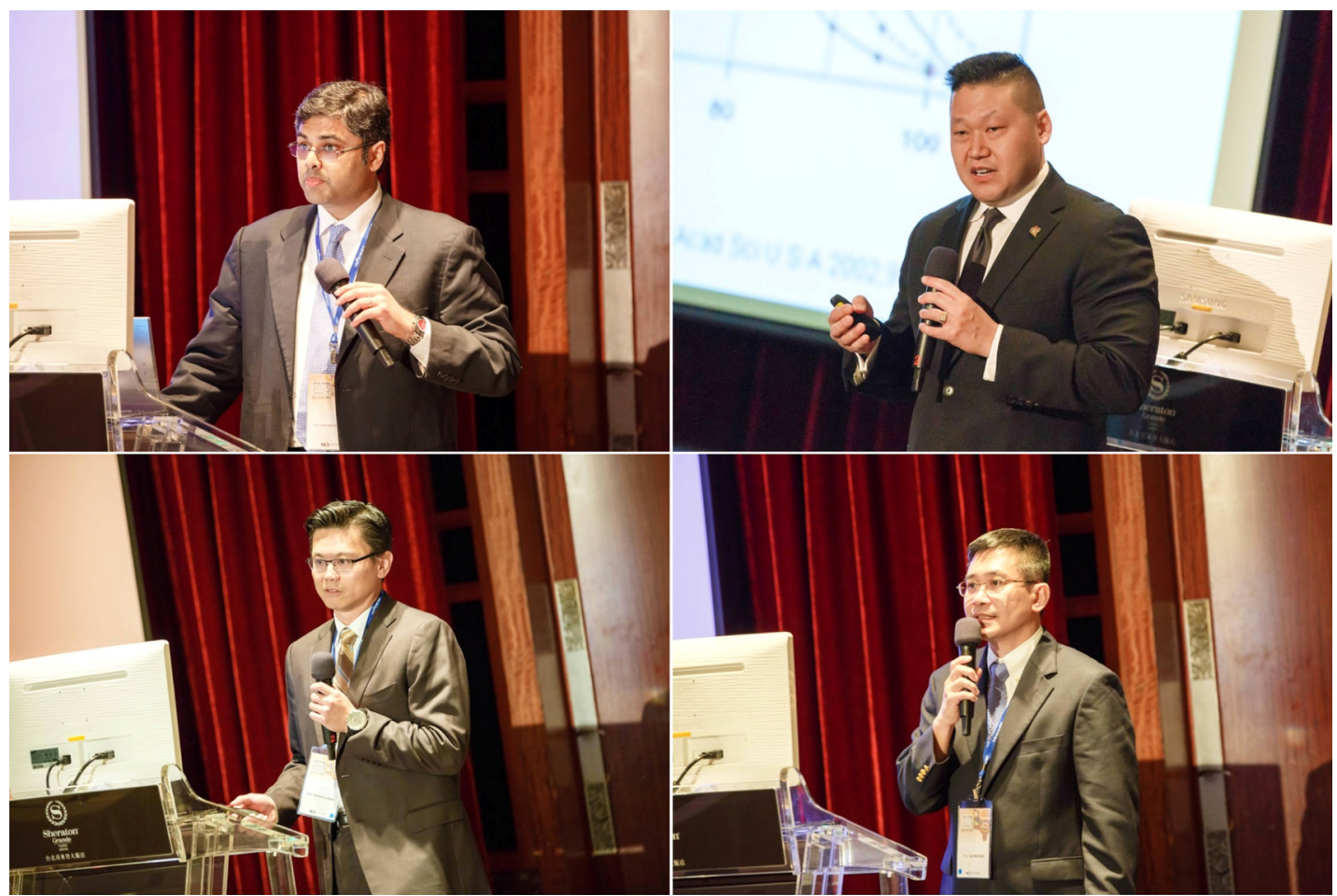

Fig. 10. Experts from all around the world in ASIA SPINE, 2015. 
with more opportunities to visit Korea and Japan to better understand local medical services.

\section{GLOBAL VISION}

In order to engage and support the global trends in spinal surgery and to fulfill our vision, many spine experts from around the world have been invited to Taiwan. For instance, Professor Praveen V. Mummaneni, the 2015-2016 Chairperson of the Association of Neurological Surgeons (AANS)/Congress of Neurological Surgeons (CNS) Section on Disorders of the Spine and Peripheral Nerves; Professor Jeffrey C. Wang, the current First Vice President of NASS and the 2012-2015 International Chairperson of Arbeitsgemeinschaft für Osteosynthesefragen Specialty Board for Spine Surgery (AOSpine); and Professor K. Daniel Riew, the 2015-2018 International Chairperson of AOSpine, were invited by President Henrich Cheng to join our 2008 annual meeting. Professor Michael Y. Wang, the 2018-2019 Chairperson of AANS/CNS Section on Disorders of the Spine and Peripheral Nerves, and Professor Fengzeng Jian, the President of the Spine and Spinal Cord Committee of the Chinese Congress of Neurosurgery, visited Taiwan for the 2009 annual meeting (Fig. 9). Professor Claudio Lamartina, Head of the Reference Center for Spinal Surgery for AOSpine European Region, visited in 2010. In 2015, President Ming-Chao Huang invited Professor Praveen V. Mummaneni, Professor Michael Y. Wang, Professor Patrick Hsieh from the University of Southern California, USA, and Professor Steven B. Kirshner from Kirshner Spine Institute, USA, to participate in the ASIA SPINE annual meeting (Fig. 10).

Neurosurgeons in Taiwan took part in international conferences as well. The 26th annual meeting of AANS/CNS Section on Disorders of the Spine and Peripheral Nerves took place in Florida in February 2010, which coincided with the handover of the 4th President, Henrich Cheng, to the 5th President, YungHsiao Chiang. The TNSS was invited by Professor Praveen V. Mummaneni, the Scientific Program Chairperson at that time, to contribute as the international society partner of this meet-
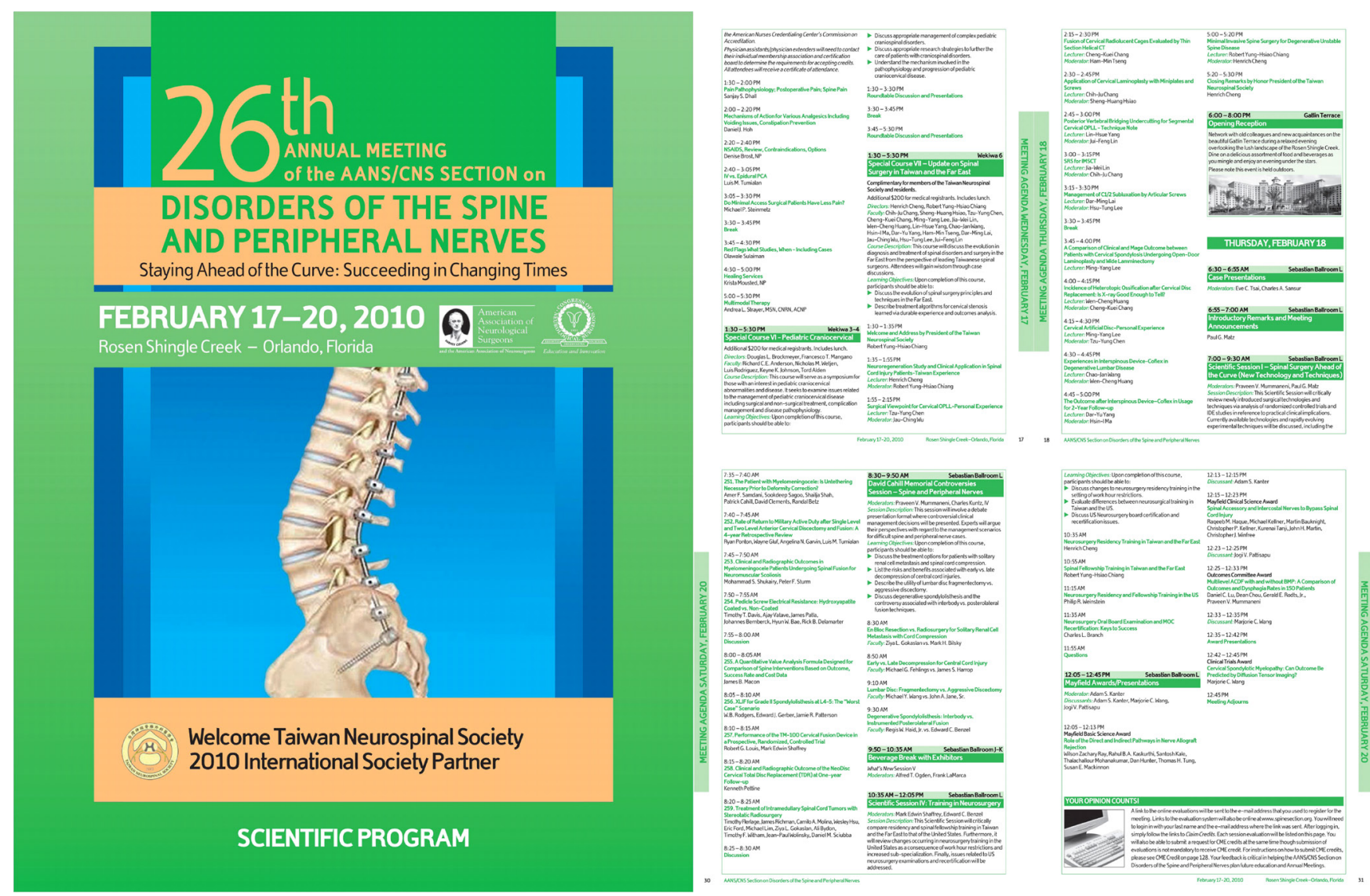

Fig. 11. Program of the 26th annual meeting of the Association of Neurological Surgeons/Congress of Neurological Surgeons section on disorders of the spine and peripheral nerves, 2010. 

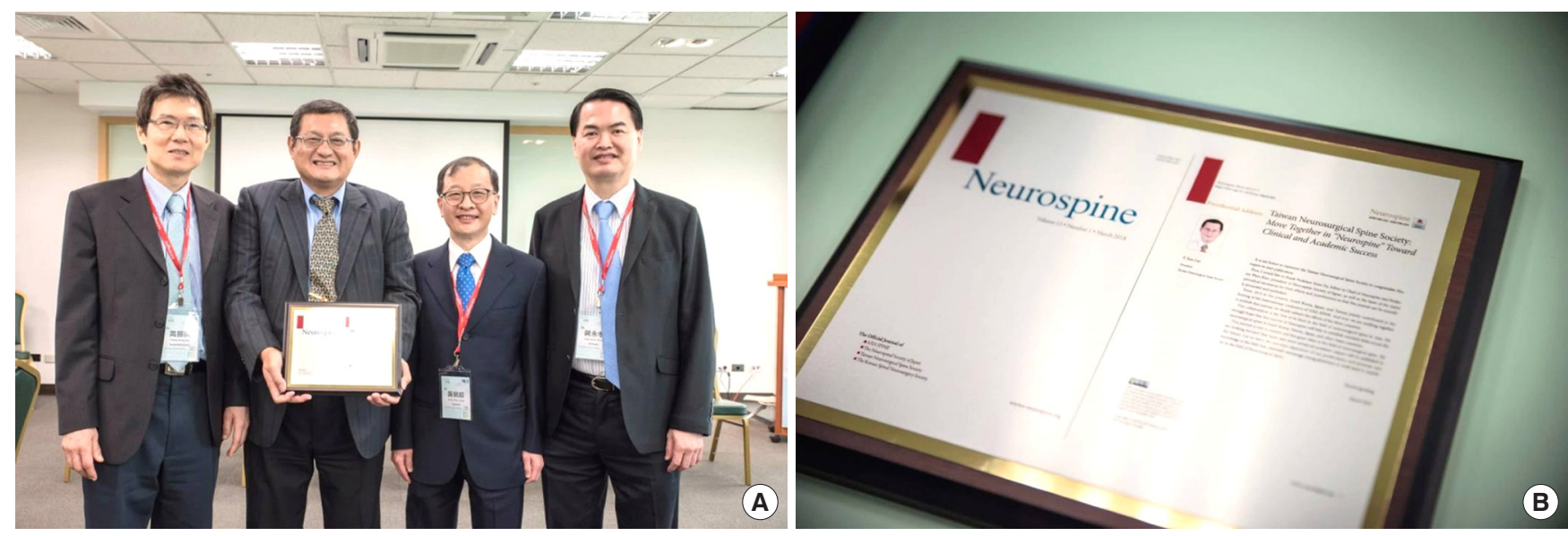

Fig. 12. (A) Presidents of the Taiwan Neurosurgical Spine Society (left to right: Cheng-Hsing Kao, E-Jian Lee, Ming-Chao Huang, and Yung-Hsiao Chiang). (B) Neurospine.

ing. In the scientific section about the training in neurosurgery on February 20, President Henrich Cheng and Yung-Hsiao Chiang spoke in front of all international experts to introduce to the world the neurosurgery residency training and spinal fellowship training, respectively, in Taiwan and the Far East. In addition, there was a special update on spinal surgery in Taiwan and the Far East, on February 17. The aim of this special meeting was to discuss the evolution in diagnosis and treatment of spinal disorders and surgery in the Far East. President Henrich Cheng and Yung-Hsiao Chiang were the course directors, and many spine specialists from Taiwan took part. Participants were: Chih-Ju Chang, Sheng-Huang Hsiao, Tzu-Yung Chen, ChengKuei Chang, Ming-Yang Lee, Jia-Wei Lin, Wen-Cheng Huang, Lin-Hsue Yang, Chao-Jan Wang, Hsin-I Ma, Dar-Yu Yang, HamMin Tseng, Dar-Ming Lai, Jau-Ching Wu, Hsu-Tung Lee, and Jui-Feng Lin (Fig. 11). All the speakers presented their faculties' achievements in English, and impressed the audience. It was a great experience for the TNSS to have the opportunity to participate in such an international conference and to develop friendships with those from overseas.

\section{PARTICIPATION IN Neurospine}

Neurospine, led by chief editor Yoon Ha, is an international peer-reviewed open-access journal, published quarterly since March 2018. The journal focuses on basic and clinical investigation of the spine and the spinal cord to enhance patient management, education, clinical or experimental research, and professionalism. As Neurospine is the official journal of ASIA SPINE, it is an honor for the TNSS to be one of the official participants of this journal. The number of published articles, hits and cross- ref citations of Neurospine increased greatly. As president E-Jian Lee said, we strongly hope that this journal will help to establish scholarly links among the professionals who deal with neurosurgical spine surgery and management in South Korea, Taiwan, Japan, and other Asian countries ${ }^{11}$ (Fig. 12).

\section{TOWARD THE FUTURE}

Since the establishment in March 2001, the TNSS has been growing, expanding and evolving step by step with efforts from all neurosurgeons in Taiwan. The TNSS is currently a group composed of 473 members, of which 367 are general members and 106 are associate members. With the greater number of members comes the greater responsibility to the development of spine surgery in Taiwan. Under President E-Jian Lee’s leadership, the TNSS was responsible for the 9th annual meeting of ASIA SPINE and the joint symposium of the TNSS, TWSS and TSMISS in 2018; there still will be some more case conferences and symposiums till the end of 2018. The spine section of resident training held by the TNSS finished successfully. In academic promotion, we joined the international peer-reviewed open-access journal Neurospine and encouraged members to publish articles. The study of spinal cord repair stepped into phase III of the clinical trial and foresaw the development of "molecular neurosurgery." As the technology and instruments advanced, new surgical techniques were introduced to our members through the exchange of knowledge.

Through neurosurgery is traditionally considered as a man's territory, there are more board-certified women neurosurgeons during these years. The current 13th President elected in 2017, Aij-Lie Kwan, is the first women President of the Taiwan Neu- 
rosurgical Society, and the first Section of Women in Neurosurgery in the annual meeting was held in the same year. As the number of women members in TNSS increases, there expectably will be more involvement of women neurosurgeon in the society works.

Being one of the major spine societies in Taiwan, the TNSS has achieved the initial goals and is still searching for more influence for spine experts and the general population. We provide a manual for spinal care on our website as a reference for patients and as a source of material for physician's health education. In addition, there is an official TNSS Facebook account sharing conference information and event highlights. Cultural elements are added into our annual meeting program as inspired by international conferences.

In the future, the TNSS will keep on expanding its territories in promoting research, holding academic seminars, participating in international conferences, and exchanging clinical experiences.

\section{CONCLUSION}

TNSS was established in 2001 and grew and bloomed till the present. As spine surgery developed in Taiwan, neurosurgeons participated in educational seminars, academic researches, scientific conferences, and international communication. With the effort of all society members, the TNSS looks forward to future successes.

\section{CONFLICT OF INTEREST}

The authors have nothing to disclose.

\section{REFERENCES}

1. Taiwan. About Taiwan [Internet]. Taipei (Taiwan): Ministry of Foreign Affairs; c2018 [cited 2018 Aug 5]. Available from: http://www.taiwan.gov.tw/about.php.

2. National Health Insurance Administration. 2017-2018 National Health Insurance Annual Report. [Internet]. Taipei (Taiwan): National Health Insurance Administration; c2015 [cited 2018 Aug 5]. Available from: http://www.nhi.gov.tw/ Content_List.aspx?n=9223A12B5B31CB37\&topn=FB01D4 69347C76A7.

3. Taiwan Medical Association. Statistics yearbook of practicing physicians and health care organizations in Taiwan, 2017 [Internet]. Taipei (Taiwan): Taiwan Medical Association; c2018 [cited 2018 Aug 5]. Available from: http://www.tma. tw/stats/index_NYearInfo.asp?/2017.html.

4. Taiwan Neurosurgical Society. History of Taiwan Neurosurgical Society [Internet]. Taipei (Taiwan): Taiwan Neurosurgical Society; c2018 [cited 2018 Aug 5]. Available from: http://www.neurosurgery.org.tw/?page_id=41.

5. Taiwan Medical Association. Lists of certified training hospitals in Taiwan, 2018 [Internet]. Taipei (Taiwan): Taiwan Medical Association; c2018 [cited 2018 Aug 5]. Available from: http://www.tma.tw/dc/dc_index.asp.

6. Wu JC, Liu L, Wen-Cheng $\mathrm{H}$, et al. The incidence of adjacent segment disease requiring surgery after anterior cervical diskectomy and fusion: estimation using an 11-year comprehensive nationwide database in Taiwan. Neurosurgery 2012;70:594-601.

7. Tu TH, Wu JC, Huang WC, et al. Heterotopic ossification after cervical total disc replacement: determination by CT and effects on clinical outcomes. J Neurosurg Spine 2011;14: 457-65.

8. Cheng H, Cao Y, Olson L. Spinal cord repair in adult paraplegic rats: partial restoration of hind limb function. Science 1996;273:510-3.

9. Journal of Neurosurgery. Editorial Board: JNS: Spine [Internet]. Charlottesvill (VA): American Association of Neurological Surgeons; c1944-2018 [cited 2018 Aug 5]. Available from: https://thejns.org/page/EB_JNSSPI/editorial-boardjns-spine.

10. Shin DA, Kim YS, Nakagawa H, et al. ASIA SPINE: the past, present, and future. Neurospine 2018;15:10-7.

11. Lee EJ. Taiwan Neurosurgical Spine Society: Move Together in "Neurospine" Toward Clinical and Academic Success. Neurospine 2018;15:7. 